\title{
Mean field expansion and meson effects in chiral condensate of analytically regularized Nambu-Jona-Lasinio model
}

\author{
R.G. Jafarov, V.E. Rochev ${ }^{\dagger}$
}

\begin{abstract}
Scalar meson contributions in chiral quark condensate are calculated in the analytically regularized Nambu - Jona-Lasinio model using the framework of mean-field expansion in bilocal-source formalism. The sigma-meson contribution for physical values of the parameters is found to be small. Pion contribution is found to be significant and should be taken into account for the choice of the parameter values.
\end{abstract}

\section{Introduction}

Nambu-Jona-Lasinio (NJL) model [1] was the first field theoretical model of dynamical chiral symmetry breaking (DCSB) in hadron physics. The NJL model has been reformulated in quark language [2] during the seventies and eighties, and since then it exists as a successful effective model of quantum chromodynamics of light hadrons in the non-perturbative region. Subsequently, the NJL model was intensively investigated also as a model of hadron matter at finite temperature and density [3]. (For more references see also reviews [4] and [5].)

In overwhelming majority of these investigations, the NJL model has been considered in the mean-field approximation (Hartree approximation), or in leading order of $1 / n_{c}$-expansion ( $n_{c}$ is the number of colors). The successes in the phenomenological applications have led to an analysis of the structure of the NJL model in next-toleading order of the expansions ( see [6]-[10] and refs. therein). Such an analysis is necessary for clarification of the region of applicability of results and the stability under a variation of parameters and quantum fluctuations due to higher-order effects.

Since the NJL model in the mean-field approximation includes quark loops, the essential aspect of application of this model is a regularization. Most common regularizations for NJL model traditionally entail a four-dimensional cutoff in Euclidean momenta or a three-dimensional momentum cutoff. Other regularization schemes (PauliVillars regularization or non-local Gauss formfactors) also are used for the NJL model. The least common regularization for NJL model is a dimensional regularization (Thus in reviews cited above, [4] and [5] this regularization is not even mentioned). At first it might appear somewhat strange, since the merits of dimensional regularization are generally known, and the same regularization most commonly used for calculations in renormalized theories, particularly in gauge theories. Apparently this fact is connected

*Physical Department of Baku State University, Baku, Azerbaijan

${ }^{\dagger}$ Institute for High Energy Physics, Protvino, Moscow region, Russia (e-mail: rochev@mx.ihep.su) 
with the following circumstance: in contrast to renormalized models, a parameter of regularization in the NJL model is included in formulae for physical quantities, and it is one of the essential parameters of the model. But the parameter of dimensional regularization, which is traditionally treated as a deviation in physical dimension of space, does not permit any physical interpretation in this treatment.

However, an alternative treatment of dimensional regularization exists - as a variant of an analytical regularization. In this treatment all calculations are made in fourdimensional Euclidean momentum space, and the regularization parameter is treated as a power of a weight function, which regularizes divergent integrals. Such treatment of dimensional regularization, based on ideas of Wilson and Collins, is consistently developed and applied to the NJL model by Krewald and Nakayama [11] in the mean-field approximation. It should be stressed that in this treatment of dimensional regularization, the regularization parameter is not at all a deviation in the physical dimension of space.

We suppose that a possible treatment of this parameter is a power of some factor, which is a measure of gluon influence on the effective four-fermion quark self-action of NJL model. In some sense this possible treatment has something in common with non-local variants of the NJL model (see [10], [12]).

In this work we study the NJL model with dimensional regularization in the treatment of Krewald and Nakayama ${ }^{1}$ in the next-to-leading order of the mean-field expansion. To formulate the mean-field expansion (Section 1) we have used an iteration scheme of solution of Schwinger-Dyson equation with fermion bilocal source, which has been developed in the literature [13]. Analytical regularization of the NJL model is discussed in Section 2. A purpose of our calculation is to study meson contributions to quark chiral condensate, which is a principal order parameter in models of DCSB. As our calculations demonstrate (Section 3), the pion contribution to chiral condensate is expressed in the analytical regularization by a very simple formula (29)- a ratio of pion contribution to leading approximation condensate is inversely proportional to the regularization parameter and does not depend on other parameters of the model. The pion contribution is rather significant, and tends to infinity at a bound of admissible values of the parameter, i.e., the model is unstable with respect to quantum fluctuations near this bound. The sigma-meson contribution is small for admissible values of the parameter. In Section 4 the results of Sections 2 and 3, which were obtained for the classical variant of the NJL model with U(1)-symmetry, are generalized for a physically interesting model with two flavors and $n_{c}$ colors ( $\mathrm{SU}(2)$-model).

A choice of physical values for $\mathrm{SU}(2)$-model parameters is discussed in Section 5. A discussion of the results is presented in the Conclusion. Quite briefly our result can be stated as follows: for physical values of parameters, the analytically regularized NJL model does not contain any pathological quantum fluctuations, connected with the scalar-meson contributions, though the pion contribution is significant and should be taken into account in phenomenological treatments of NJL-type models.

\footnotetext{
${ }^{1}$ To avoid unnecessary associations with the usual treatment of dimensional regularization we shall in what follows, refer to this regularization as analytical regularization of NJL model
} 


\section{Mean-Field Expansion in Bilocal-Source Ap- proach}

By the U(1)-model, we mean a theory of self interacting spinor field $\psi$ defined by the Lagrangian

$$
\mathcal{L}=\bar{\psi} i \hat{\partial} \psi+\frac{g}{2}\left((\bar{\psi} \psi)^{2}+\left(\bar{\psi} i \gamma_{5} \psi\right)^{2}\right) .
$$

Here $g>0$ is a coupling. This Lagrangian is invariant under transformations of the chiral group $U_{V}(1) \times U_{A}(1)$. Such a model has no direct physical applications, but, as is shown in Section 4, the results of physical $\mathrm{SU}(2)$-model for meson contributions in chiral condensate are practically identical to the results of the U(1)-model. This is not surprising since these contributions are purely dynamical. Distinctions are displayed as simple coefficients (see Eq. (40) of Section 4).

A generating functional of Green functions (vacuum expectation values of $T$-products of fields) can be represented as the functional integral with bilocal source

$$
G(\eta)=\int D(\psi, \bar{\psi}) \exp i\left\{\int d x \mathcal{L}-\int d x d y \bar{\psi}(y) \eta(y, x) \psi(x)\right\},
$$

where $\eta(y, x)$ is the bilocal source of the spinor field.

The functional derivative of $G$ over source $\eta$ is a one-particle (two-point) Green function (a propagator of the field $\psi$ ):

$$
\left.\frac{\delta G}{\delta \eta(y, x)}\right|_{\eta=0}=i<0|T\{\psi(x) \bar{\psi}(y)\}| 0>\equiv S(x-y) .
$$

The $n$-th functional derivative of $G$ over source $\eta$ is the $n$-particle (2n-point) Green function:

$\left.\frac{\delta^{n} G}{\delta \eta\left(y_{1}, x_{1}\right) \cdots \delta \eta\left(y_{n}, x_{n}\right)}\right|_{\eta=0}=i^{n}<0\left|T\left\{\psi\left(x_{1}\right) \bar{\psi}\left(y_{1}\right) \cdots \psi\left(x_{n}\right) \bar{\psi}\left(y_{n}\right)\right\}\right| 0>\equiv S_{n}\left(\begin{array}{cc}x_{1} & y_{1} \\ \cdots & \cdots \\ x_{n} & y_{n}\end{array}\right)$.

Translational invariance of the functional-integration measure in Eq. (2) give us the functional-differential Schwinger-Dyson equation (SDE) for the generating functional $G$ :

$$
\begin{aligned}
& \delta(x-y) G+i \hat{\partial}_{x} \frac{\delta G}{\delta \eta(y, x)}+i g\left\{\frac{\delta}{\delta \eta(y, x)} \operatorname{tr} \frac{\delta G}{\delta \eta(x, x)}-\gamma_{5} \frac{\delta}{\delta \eta(y, x)} \operatorname{tr} \gamma_{5} \frac{\delta G}{\delta \eta(x, x)}\right\}= \\
&=\int d x_{1} \eta\left(x, x_{1}\right) \frac{\delta G}{\delta \eta\left(y, x_{1}\right)}
\end{aligned}
$$

We shall solve this equation employing the method proposed in [13]. (See also the brief review in [14].)

For the NJL model this method is a variant of the mean-field expansion.

A leading approximation is an approximation of the functional-differential SDE (4) by the equation with zero r.h.s:

$$
\delta(x-y) G^{(0)}+i \hat{\partial}_{x} \frac{\delta G^{(0)}}{\delta \eta(y, x)}+i g\left\{\frac{\delta}{\delta \eta(y, x)} \operatorname{tr} \frac{\delta G^{(0)}}{\delta \eta(x, x)}-\gamma_{5} \frac{\delta}{\delta \eta(y, x)} \operatorname{tr} \gamma_{5} \frac{\delta G^{(0)}}{\delta \eta(x, x)}\right\}=0
$$

A solution of Eq.(5) is the functional

$$
G^{(0)}=\exp \left\{\operatorname{Tr}\left(S^{(0)} * \eta\right)\right\}
$$


(here $\operatorname{Tr}$ is a trace in operator sense, and $*$ is operator multiplication), where $S^{(0)}$ is a solution of equation

$$
\delta(x)+i \hat{\partial} S^{(0)}(x)+i g\left\{S^{(0)}(x) \operatorname{tr} S^{(0)}(0)-\gamma_{5} S^{(0)}(x) \operatorname{tr} \gamma_{5} S^{(0)}(0)\right\}=0 .
$$

Leading approximation (5)-(6) generates the linear iteration scheme

$$
G=G^{(0)}+G^{(1)}+\cdots+G^{(n)}+\cdots,
$$

where $n$-th step functional $G^{(n)}$ is a solution of the equation

$$
G^{(n)}+i \hat{\partial} \frac{\delta G^{(n)}}{\delta \eta}+i g\left\{\frac{\delta}{\delta \eta} \operatorname{tr} \frac{\delta G^{(n)}}{\delta \eta}-\gamma_{5} \frac{\delta}{\delta \eta} \operatorname{tr} \gamma_{5} \frac{\delta G^{(n)}}{\delta \eta}\right\}=\eta * \frac{\delta G^{(n-1)}}{\delta \eta} .
$$

A solution of Eq.(8) is the functional

$$
G^{(n)}=P^{(n)} G^{(0)},
$$

where $P^{(n)}$ is a polynomial of $2 n$-th degree on source $\eta$.

It follows from (6), that the unique connected Green function of the leading approximation is the propagator $S^{(0)}$. Other connected Green functions appear in the following iteration steps.

A solution of equation (7) is the free propagator

$$
S^{(0)}(p)=\frac{1}{m-\hat{p}}
$$

with dynamical mass $m$, which is a solution of the gap equation of the NJL model:

$$
m=-4 i g m \int \frac{d \tilde{p}}{m^{2}-p^{2}} .
$$

Here and below $d \tilde{p} \equiv d^{4} p /(2 \pi)^{4}$.

The divergent integral in the r.h.s. of equation (9) should be dealt with by some regularization. The chiral-symmetric, trivial solution $m=0$, always exists. Physically preferable (energetically favorable) solution is a solution with $m \neq 0$, which corresponds to DCSB. Below we shall consider just such a solution.

The first iteration is the functional

$$
G^{(1)}=\left\{\frac{1}{2} \operatorname{Tr}\left(S_{2}^{(1)} * \eta^{2}\right)+\operatorname{Tr}\left(S^{(1)} * \eta\right)\right\} G^{(0)} .
$$

Taking into account Eqs.(5)-(8), we obtain the first iteration equation for the twoparticle function

$$
\begin{gathered}
S_{2}^{(1)}\left(\begin{array}{cc}
x & y \\
x^{\prime} & y^{\prime}
\end{array}\right)=-S^{(0)}\left(x-y^{\prime}\right) S^{(0)}\left(x^{\prime}-y\right)+ \\
+i g \int d x_{1}\left\{\left(S^{(0)}\left(x-x_{1}\right) S^{(0)}\left(x_{1}-y\right)\right) \operatorname{tr} S_{2}^{(1)}\left(\begin{array}{cc}
x_{1} & x_{1} \\
x^{\prime} & y^{\prime}
\end{array}\right)-\right. \\
\left.-\left(S^{(0)}\left(x-x_{1}\right) \gamma_{5} S^{(0)}\left(x_{1}-y\right)\right) \operatorname{tr} \gamma_{5} S_{2}^{(1)}\left(\begin{array}{cc}
x_{1} & x_{1} \\
x^{\prime} & y^{\prime}
\end{array}\right)\right\}
\end{gathered}
$$


and the equation for the first iteration corrections to the propagator

$$
\begin{gathered}
S^{(1)}(x-y)=i g \int d x_{1} S^{(0)}\left(x-x_{1}\right)\left\{S_{2}^{(1)}\left(\begin{array}{cc}
x_{1} & y \\
x_{1} & x_{1}
\end{array}\right)-\gamma_{5} S_{2}^{(1)}\left(\begin{array}{cc}
x_{1} & y \\
x_{1} & x_{1}
\end{array}\right) \gamma_{5}\right\}+ \\
+i g \int d x_{1} S^{(0)}\left(x-x_{1}\right) S^{(0)}\left(x_{1}-y\right) \operatorname{tr} S^{(1)}(0) .
\end{gathered}
$$

A solution of the linear integral equation (10) is

$$
\begin{gathered}
S_{2}^{(1)}\left(\begin{array}{cc}
x & y \\
x^{\prime} & y^{\prime}
\end{array}\right)= \\
=\int d x_{1} d y_{1} d x_{1}^{\prime} d y_{1}^{\prime} S^{(0)}\left(x-x_{1}\right) S^{(0)}\left(x^{\prime}-x_{1}^{\prime}\right) F_{2}\left(\begin{array}{cc}
x_{1} & y_{1} \\
x_{1}^{\prime} & y_{1}^{\prime}
\end{array}\right) S^{(0)}\left(y_{1}-y\right) S^{(0)}\left(y_{1}^{\prime}-y^{\prime}\right),
\end{gathered}
$$

where the amputated two-particle function $F_{2}$ is

$$
\begin{gathered}
F_{2}\left(\begin{array}{cc}
x & y \\
x^{\prime} & y^{\prime}
\end{array}\right)=-\left[S^{(0)}\right]^{-1}\left(x-y^{\prime}\right)\left[S^{(0)}\right]^{-1}\left(x^{\prime}-y\right)+ \\
+\delta(x-y) \delta\left(x^{\prime}-y^{\prime}\right)\left\{1 \otimes 1 \cdot A_{\sigma}\left(x-x^{\prime}\right)+\gamma_{5} \otimes \gamma_{5} \cdot A_{\pi}\left(x-x^{\prime}\right)\right\},
\end{gathered}
$$

and scalar amplitudes $A_{\sigma}$ and $A_{\pi}$ in momentum space are

$$
A_{\sigma}(p)=-\frac{i g}{1-L_{S}(p)}, \quad L_{S}(p)=i g \int d \tilde{q} \operatorname{tr} S^{(0)}(p+q) S^{(0)}(q)
$$

and

$$
A_{\pi}(p)=\frac{i g}{1+L_{P}(p)}, \quad L_{P}(p)=i g \int d \tilde{q} \operatorname{tr} S^{(0)}(p+q) \gamma_{5} S^{(0)}(q) \gamma_{5} .
$$

Taking into account the gap equation (9) with $m \neq 0$, one can obtain (in translationalinvariant regularization) for $A_{\sigma}$ and $A_{\pi}$ the following representations:

$$
\begin{gathered}
A_{\sigma}(p)=\frac{1}{2\left(4 m^{2}-p^{2}\right) I_{0}(p)} \\
A_{\pi}(p)=\frac{1}{2 p^{2} I_{0}(p)} .
\end{gathered}
$$

Here

$$
I_{0}(p)=\int d \tilde{q} \frac{1}{\left(m^{2}-(p+q)^{2}\right)\left(m^{2}-q^{2}\right)}
$$

Eq.(11) for $S^{(1)}$, taking into account the results for $S_{2}^{(1)}$, reduces in the momentum space to a system of simple algebraic equations. Introducing the first iteration mass operator $\Sigma^{(1)}=\left[S^{(0)}\right]^{-1} \star S^{(1)} \star\left[S^{(0)}\right]^{-1}$ we obtain from equation (11):

$$
\Sigma^{(1)}(x)=i g \delta(x) \operatorname{tr} S^{(1)}(0)+S^{(0)}(x) A_{\sigma}(x)+S^{(0)}(-x) A_{\pi}(x) .
$$

Let us briefly discuss the following step of the iteration scheme. A solution of the second iteration functional-derivative equation has the form

$$
G^{(2)}=\left\{\frac{1}{4 !} \operatorname{Tr}\left(S_{4}^{(2)} * \eta^{4}\right)+\frac{1}{3 !} \operatorname{Tr}\left(S_{3}^{(2)} * \eta^{3}\right)+\frac{1}{2} \operatorname{Tr}\left(S_{2}^{(2)} * \eta^{2}\right)+\operatorname{Tr}\left(S^{(2)} * \eta\right)\right\} G^{(0)},
$$

i.e., in the second iteration equations a four-particle and three-particle functions $\left(S_{4}^{(2)}\right.$ and $S_{3}^{(2)}$ ) appear. Equations for $S_{2}^{(2)}$ and $S^{(2)}$ have the same form as for the first iteration, except for inhomogeneous terms, which contain $S_{4}^{(2)}$ and $S_{3}^{(2)}$ for the second iteration equations. 


\section{Dimensional regularization in the NJL model}

Due to non-renormalizability of the NJL model, the regularization is an essential component of the model.

We shall use in this work the dimensional regularization in a variant form, which was proposed in [11]. The general principles of the approach of [11] are the following:

- All calculations are made in 4-dimensional Euclidean space;

- Translational invariance is assumed;

- The regularization procedure consists in transformation of integration measure by a weight function which provides a convergence of the integrals.

In this approach the dimensional regularization is, in essence, a variant of analytical regularization. This point is very important for the use and interpretation of this regularization. In this connection we shall, in what follows, use the term "analytical" for this regularization to stress its peculiarities in contrast to the usual treatment of the dimensional regularization in a formal D-dimensional space. Let us consider the gap equation (9) of the NJL model as an example.

The gap equation with $m \neq 0$ in Euclidean space after angle integration is

$$
1=2 g \frac{\Omega_{4}}{(2 \pi)^{4}} \int \frac{q_{e}^{2} d q_{e}^{2}}{m^{2}+q_{e}^{2}},
$$

where $\Omega_{4}=2 \pi^{2}$ is a surface of 4 -dimensional unit sphere. In accordance with the previous comment, we modify the integrand by the weight function:

$$
w_{\Lambda, D}\left(q_{e}^{2}\right)=w_{\Lambda}\left(q_{e}^{2}\right) w_{D}\left(q_{e}^{2}\right)=\theta\left(\Lambda^{2}-q_{e}^{2}\right)\left(\frac{\mu^{2}}{q_{e}^{2}}\right)^{2-D / 2} .
$$

The weight function $w_{\Lambda, D}$ is the product of two weight functions $w_{\Lambda}$ and $w_{D}$. The function $w_{\Lambda}$ corresponds to the four dimensional cutoff regularization, while the function $w_{D}$ corresponds to dimensional (analytical) regularization.

A calculation of the integral over $d q_{e}^{2}$ results in

$$
1=\frac{2 g m^{2} \Omega_{4}}{(2 \pi)^{4}}\left(\frac{m^{2}}{\mu^{2}}\right)^{D / 2-2} B_{\frac{\Lambda^{2}}{m^{2}+\Lambda^{2}}}(D / 2,1-D / 2) .
$$

Here $B_{x}(u, v)$ is the incomplete Beta-function.

a) Cutoff: By setting $D=4$, we have

$$
1=\kappa_{\Lambda}\left(1-\frac{m^{2}}{\Lambda^{2}} \log \left(1+\frac{\Lambda^{2}}{m^{2}}\right)\right)
$$

where $\kappa_{\Lambda}=g \Lambda^{2} / 4 \pi^{2}$.

This relation coincides exactly with the classical result of [1].

b) Analytical (dimensional) regularization: When $\Lambda^{2} \rightarrow \infty$, taking into account the formula

$$
B_{1}(D / 2,1-D / 2)=\Gamma(D / 2) \Gamma(1-D / 2)
$$

and rescaling the scale parameter $\mu^{2}$ as

$$
\left(\mu^{2}\right)^{2-D / 2}=\frac{\Omega_{D}}{\Omega_{4}} \frac{(2 \pi)^{4}}{(2 \pi)^{D}}(M)^{2-D / 2}
$$


we obtain for the gap equation

$$
1=\kappa \Gamma(1-D / 2)\left(\frac{m^{2}}{4 \pi M^{2}}\right)^{D / 2-2} .
$$

Here instead of $g$ we introduce the dimensionless quantity

$$
\kappa=\frac{g m^{2}}{4 \pi^{2}} \text {. }
$$

Equation (21) corresponds exactly to the calculation of the initial integral with the formal prescription of D-dimensional integration

$$
d \tilde{q} \equiv \frac{d^{4} q}{(2 \pi)^{4}} \rightarrow \frac{\left(M^{2}\right)^{2-D / 2} d^{D} q}{(2 \pi)^{D}}
$$

but in our case the calculation was performed in the usual 4-dimensional space, i.e. in our treatment $D$ is not a dimension of some space, but a parameter which provides the convergence. In particular, we are not constrained by the limit $D \rightarrow 4$ for the analysis of the results. We suppose that a possible treatment of regularization parameter is a power of some additional factor, which is a measure of gluon influence on the effective local four-quark self-interaction of the NJL model.

Below we shall use the regularization parameter $\xi$ as ${ }^{2}$

$$
D=2-2 \xi
$$

In terms of the parameter $\xi$ gap equation (21) has the form

$$
1=\kappa \Gamma(\xi)\left(\frac{4 \pi M^{2}}{m^{2}}\right)^{1+\xi}
$$

The region of convergence of the integral is $0<\xi<1$. As we shall see (Section 5), this is also the region for the physical values of the model parameters.

Chiral condensate in the leading approximation is

$$
\left(c^{3}\right)^{(0)}=i \operatorname{tr} S^{(0)}(0)=-\frac{m^{3}}{4 \pi^{2}}\left(\frac{4 \pi M^{2}}{m^{2}}\right)^{1+\xi} \Gamma(\xi)=-\frac{m}{g} .
$$

Integral $I_{0}$ (see (18)), which is a part of scalar amplitudes $A_{\sigma}$ and $A_{\pi}$, can also be calculated as above. Transforming to Euclidean metric, introducing a standard Feynman parameterization, and changing an integration variable (which is possible due to translational invariance of the procedure, see [11]) we can perform the angular integration. According to the procedure, we introduce into the integrand, a weight function $w_{D}\left(q_{e}^{2}\right)$ and, after the same rescaling (20), obtain the result, which also exactly corresponds to the result of integration with the formal transition to $D$-dimensional space.

Taking into account the gap equation (24), we obtain the pole approximation for the scalar amplitudes in analytical regularization

$$
A_{\sigma}(p) \simeq \frac{1}{2\left(4 m^{2}-p^{2}\right) I_{0}\left(4 m^{2}\right)}=\frac{2 i g m^{2}(1+2 \xi)}{\left(4 m^{2}-p^{2}\right) \xi},
$$

\footnotetext{
${ }^{2}$ Note that parameter $\xi$ is different from the commonly used parameter $\varepsilon=(4-D) / 2$. They are connected by the relation $\varepsilon=1+\xi$. Introduction of this notation prevents unnecessary associations with the usual treatment of dimensional regularization. Furthermore, in terms of the parameter $\xi$ all subsequent formulae of the NJL model acquire simple forms.
} 


$$
A_{\pi}(p) \simeq \frac{1}{2 p^{2} I_{0}(0)}=-\frac{2 i g m^{2}}{p^{2} \xi}
$$

which correspond to contributions of sigma-meson and pion. We shall use these expressions in the following calculations.

\section{Meson contributions to the chiral condensate}

As a measure of quantum fluctuations of the chiral field, consider a ratio of first iteration condensate

$$
\left(c^{3}\right)^{(1)}=i \operatorname{tr} S^{(1)}(0)
$$

to the leading-approximation condensate $\left(c^{3}\right)^{(0)}$ :

$$
r \equiv \frac{\left(c^{3}\right)^{(1)}}{\left(c^{3}\right)^{(0)}}=r_{\sigma}+r_{\pi}
$$

Here $r_{\sigma}$ is a contribution of the scalar meson (sigma-meson) and $r_{\pi}$ is a contribution of the pseudoscalar meson (pion).

From Eq.(19), we obtain

$$
r_{\sigma}=-\frac{2 i g}{\xi} \int \frac{d \tilde{p} d \tilde{q}\left(m^{2}+3 p^{2}-2(p q)\right)}{\left(m^{2}-p^{2}\right)^{2}\left(m^{2}-(p-q)^{2}\right)} A_{\sigma}(q),
$$

and

$$
r_{\pi}=-\frac{2 i g}{\xi} \int \frac{d \tilde{p} d \tilde{q}\left(m^{2}-p^{2}+2(p q)\right)}{\left(m^{2}-p^{2}\right)^{2}\left(m^{2}-(p-q)^{2}\right)} A_{\pi}(q) .
$$

Calculation of these two-loop integrals gives us the desired answer.

The integrals for $r_{\pi}$ are calculated in the analytical regularization in closed form and give us a very simple expression

$$
r_{\pi}=\frac{1}{4 \xi}
$$

The scalar contribution can be represented as

$$
\begin{gathered}
r_{\sigma}=\frac{4^{\xi} \Gamma\left(\frac{3}{2}+\xi\right)}{2 \sqrt{\pi} \Gamma(3+\xi)} \int_{0}^{1} \frac{d u}{(4-3 u)^{1+\xi}}\left[(3-2 u)(1-\xi) F\left(1+\xi, 2-\xi ; 3+\xi ; \frac{(u-2)^{2}}{4-3 u}\right)-\right. \\
\left.-(1+2 \xi) F\left(1+\xi, 1-\xi ; 3+\xi ; \frac{(u-2)^{2}}{4-3 u}\right)\right]
\end{gathered}
$$

where $F(a, b ; c ; z)$ is the Gauss hypergeometric function.

Note, that $r_{\pi}$ and $r_{\sigma}$ depend only on the parameter $\xi$ and do not depend on other parameters of the model. This feature is a peculiarity of the analytical regularization.

Results of the calculations in the region $0<\xi \leq 1$ are shown in Table 1. One can see that the contribution of the sigma-meson is small, while the contribution of the pion in the region $0<\xi \leq 0.3 \div 0.5$ is significant and, strictly speaking, in this last region we cannot consider this contribution as a mere correction, i.e., at such values of the parameter $\xi$ the quantum fluctuations are large and can lead to a principal modification of the entire physical picture of the NJL model (in spirit of work [15], for example). 


\begin{tabular}{c|ccc}
\hline$\xi$ & $r_{\sigma}$ & $r_{\pi}$ & $c^{(1)} / c^{(0)}$ \\
\hline \hline 0.1 & 0.264 & 2.50 & 0.556 \\
0.2 & 0.189 & 1.250 & 0.346 \\
0.3 & 0.119 & 0.833 & 0.250 \\
0.4 & 0.057 & 0.625 & 0.189 \\
0.5 & 0 & 0.50 & 0.145 \\
0.6 & -0.050 & 0.417 & 0.110 \\
0.7 & -0.094 & 0.357 & 0.081 \\
0.8 & -0.131 & 0.313 & 0.057 \\
0.9 & -0.162 & 0.278 & 0.037 \\
1.0 & -0.188 & 0.250 & 0.021 \\
\hline
\end{tabular}

Table 1: Relative contributions of sigma-meson $\left(r_{\sigma}\right)$ and pion $\left(r_{\pi}\right)$ to the chiral condensate $c^{3}$ of $\mathrm{U}(1)$-model and a ratio $\left(c^{(1)} / c^{(0)}\right)$ of first iteration condensate to leading-approximation condensate as functions of the regularization parameter $\xi$.

\section{$5 \quad \mathrm{SU}(2)$-model}

The U(1)-model has no direct physical applications, and we cannot estimate the physical values of model parameters in the framework of this model and, thus cannot reach any definite conclusion about the meson contributions.

To study the possible values of the parameters $m, \kappa$ and $\xi$, let us consider a model connected with light hadron phenomenology. Accordingly we study the model defined by the Lagrangian

$$
\mathcal{L}=\bar{\psi} i \hat{\partial} \psi+\frac{g}{2}\left[(\bar{\psi} \psi)^{2}+\left(\bar{\psi} i \gamma_{5} \tau^{a} \psi\right)^{2}\right] .
$$

Here $\psi \equiv \psi_{j}^{\alpha, c} ; \quad \alpha=1,2,3,4$ is Dirac spinor index; $c=1, \ldots, n_{c}$ is color index; $j=1,2$ is isotopic (flavor) index; $\tau^{a}$ are generators of $S U(2)$ (Pauli matrices); $a=1,2,3$. This model has the chiral symmetry of $S U_{V}(2) \times S U_{A}(2)$-group. We call this the $\mathrm{SU}(2)$ model.

Mean-field expansion in bilocal-source formalism is constructed with the same scheme as in the U(1)-model above, and so without going to the details, we enumerate only the major differences from corresponding results of U(1)-model.

In the leading approximation, the propagator is diagonal over color and flavor:

$$
S_{c d, j k}^{(0)}=\delta_{c d} \delta_{j k}(m-\hat{p})^{-1},
$$

and the gap equation for the $\mathrm{SU}(2)$-model is

$$
1=-8 i g n_{c} \int \frac{d \tilde{p}}{m^{2}-p^{2}} .
$$

The chiral condensate is defined for each flavor, i.e.,

$$
c_{u}^{3}=<0|\bar{u} u| 0>, \quad c_{d}^{3}=<0|\bar{d} d| 0>.
$$

(In the chiral limit $c_{d}=c_{u}$.) Two-particle first iteration amplitude $A$ (connected part of amputated two-particle function $S_{2}^{(1)}$ ) has the following color and flavor structure:

$$
A_{c^{\prime} d^{\prime}, j^{\prime} k^{\prime}}^{c d, j k}=\delta^{c d} \delta^{c^{\prime} d^{\prime}}\left[\delta_{j k} \delta_{j^{\prime} k^{\prime}} A_{\sigma}+\tau_{j k}^{a} \tau_{j^{\prime} k^{\prime}}^{a} A_{\pi}\right] .
$$


Scalar amplitudes $A_{\sigma}$ and $A_{\pi}$ are defined by formulae (14) and (15), but traces in the definitions of loops $L_{S}$ and $L_{P}$ are now taken over all discrete indices.

First iteration mass operator $\Sigma^{(1)}=\left[S^{(0)}\right]^{-1} \star S^{(1)} \star\left[S^{(0)}\right]^{-1}$ is diagonal over color and flavor, and is connected with the scalar amplitudes by relation

$$
\Sigma^{(1)}(x)_{j k}^{c d}=\delta^{c d} \delta_{j k} \cdot\left[i g \delta(x) \operatorname{tr} S^{(1)}(0)+S^{(0)}(x) A_{\sigma}(x)+3 S^{(0)}(-x) A_{\pi}(x)\right] .
$$

For the ratio of the first iteration condensate to the leading-approximation condensate we obtain

$$
\begin{gathered}
r=r_{\sigma}+r_{\pi}= \\
=-\frac{8 i g n_{c}}{1-8 i g n_{c} J} \int \frac{d \tilde{q} d \tilde{p}}{\left(m^{2}-p^{2}\right)^{2}\left(m^{2}-(p-q)^{2}\right)}\left(\left(3 p^{2}-2(p q)+m^{2}\right) A_{\sigma}(q)+3\left(m^{2}-p^{2}+2(p q)\right) A_{\pi}(q)\right),
\end{gathered}
$$

where

$$
J=\int d \tilde{p} \frac{m^{2}+p^{2}}{\left(m^{2}-p^{2}\right)^{2}} .
$$

The gap equation in analytical regularization for the $\mathrm{SU}(2)$-model has exactly same form as in (24), if we redefine the dimensionless constant $\kappa$ as

$$
\kappa=\frac{g n_{c} m^{2}}{2 \pi^{2}}=2 n_{c} \kappa_{0}
$$

(Here and henceforward, the index 0 denotes corresponding quantities of the U(1)model.)

The scalar amplitudes of SU(2)-model can be written as

$$
\begin{gathered}
A_{\sigma}=\frac{1}{4 n_{c}\left(4 m^{2}-p^{2}\right) I_{0}}, \\
A_{\pi}=\frac{1}{4 n_{c} p^{2} I_{0}},
\end{gathered}
$$

where $I_{0}$ is defined by formula (18).

Taking into account the above relations and definitions we obtain for the meson contributions of the $\mathrm{SU}(2)$-model:

$$
r_{\sigma}=\frac{1}{2 n_{c}} r_{0 \sigma}, \quad r_{\pi}=\frac{3}{2 n_{c}} r_{0 \pi},
$$

where $r_{0 \sigma}$ and $r_{0 \pi}$ are corresponding contributions of the $\mathrm{U}(1)$-model.

For physical values of colors $n_{c}=3$, both contributions decrease compared to the corresponding contributions of the $\mathrm{U}(1)$-model: $r_{\sigma}$ to six times, and $r_{\pi}$ to half. Accordingly the bounds of the region of large fluctuations are moved. Results of the calculations in region $0<\xi \leq 1$ are shown in Table 2 . We see, that for the $\mathrm{SU}(2)$ model at $\xi \geq 0.2$ the first iteration condensate $c^{(1)}$ is not more than $20 \%$ different from the leading-approximation condensate, i.e., we may consider these values of the regularization parameter to be in the stability zone with respect to fluctuations caused by the meson contributions. 


\begin{tabular}{c|ccc}
\hline$\xi$ & $r_{\sigma}$ & $r_{\pi}$ & $c^{(1)} / c^{(0)}$ \\
\hline \hline 0.1 & 0.044 & 1.250 & 0.319 \\
0.2 & 0.032 & 0.625 & 0.183 \\
0.3 & 0.020 & 0.417 & 0.128 \\
0.4 & 0.010 & 0.313 & 0.098 \\
0.5 & 0 & 0.250 & 0.077 \\
0.6 & -0.008 & 0.209 & 0.063 \\
0.7 & -0.016 & 0.179 & 0.052 \\
0.8 & -0.022 & 0.157 & 0.043 \\
0.9 & -0.027 & 0.139 & 0.036 \\
1.0 & -0.031 & 0.125 & 0.030 \\
\hline
\end{tabular}

Table 2: Relative contributions of sigma-meson $\left(r_{\sigma}\right)$ and pion $\left(r_{\pi}\right)$ to chiral condensate $c^{3}$ of $\mathrm{SU}(2)$-model and ratio of first-step condensate to leading-approximation condensate $\left(c^{(1)} / c^{(0)}\right)$ as functions of regularization parameter $\xi$.

\section{Choice of parameters}

In determining the $\mathrm{SU}(2)$-model parameters (dynamical quark mass $m$, regularization parameter $\xi$ and coupling $g$ (or dimensionless constant $\left.\kappa=g n_{c} m^{2} / 2 \pi^{2}\right)$ ), it is necessary to connect the values of the parameters with measurable quantities. We accordingly choose the values of the pion decay constant, the chiral condensate and the decay width of $\pi^{0}$-meson into two photons: $\pi^{0} \rightarrow \gamma \gamma$.

Pion decay constant $f_{\pi}=93 \mathrm{MeV}$ is defined by

$$
i \delta^{a b} k_{\mu} f_{\pi}=<0\left|\bar{\psi} \gamma_{\mu} \gamma_{5} \frac{\tau^{a}}{2} \psi\right| b, k>
$$

where $\mid b, k>$ is a pion state $b$ with momentum $k_{\mu}$.

For the $\mathrm{SU}(2)$-model the following formula for $f_{\pi}$ (see, for example, [4]):

$$
f_{\pi}^{2}=-4 i n_{c} m^{2} I_{0}(0)
$$

is regularization-independent. In the analytical regularization we have $I_{0}(0)=i \xi / 16 \pi^{2} \kappa$ and obtain:

$$
f_{\pi}^{2}=\frac{\xi}{2 g}
$$

The leading-approximation chiral condensate is

$$
c=(<0|\bar{\psi} \psi| 0>/ 2)^{1 / 3}=-(m / 2 g)^{1 / 3},
$$

which is regularization-independent. Decay width $\Gamma_{\pi^{0} \gamma \gamma}=7.7 \mathrm{KeV}$ has been calculated in the analytical regularization for $\mathrm{SU}(2)$-model in ref.[11]. In our notation, this formula has the form

$$
\Gamma_{\pi^{0} \gamma \gamma}=\frac{\alpha^{2} m_{\pi}^{3} \xi^{2}(1+\xi)^{2}}{64 \pi^{3} f_{\pi}^{2} \kappa^{2}}
$$

Here $\alpha=1 / 137$ is the fine structure, $m_{\pi}=135 \mathrm{MeV}$ is the mass of $\pi^{0}$-meson.

Formulae (43)-(45) enable us to determine the SU(2)-model parameters.

Chiral condensate $c=(\langle\bar{\psi} \psi\rangle / 2)^{1 / 3}$ is not a directly measurable quantity. We determine the parameter values for some typical values of the chiral condensate. 
At $c=-160 \mathrm{MeV}$ we have $\xi \cong 1, m \cong 475 \mathrm{MeV}, \kappa \cong 2$. Note, that for this value of regularization parameter $\xi$, the correction to condensate $c$ is about $3 \%$ (see Table 2), i.e. the model is stable with respect to quantum fluctuations caused by the mesons. On the other hand, such a low value of the chiral condensate hardly agrees with phenomenology, since the Gell-Mann-Oakes-Renner formula leads to large values of current quark masses. At $c=-200 \mathrm{MeV}$ we obtain $\xi \cong 0.44, m \cong 400$ $\mathrm{MeV}, \kappa \cong 0.62$. The correction to condensate $c$ is $9 \%$. At $c=-250 \mathrm{MeV}$, we obtain $\xi \cong 0.2, m \cong 370 \mathrm{MeV}, \kappa \cong 0.24$, and the correction to condensate $c$ is more then $18 \%$. Thus fixing the model parameters with formulae (43)-(45) at phenomenologically acceptable condensate values $c=-(200 \div 250) \mathrm{MeV}$ corresponds to the condensate corrections of the order of $10 \div 20 \%$.

The model parameters as chosen above, were implemented in the leading-approximation formula (44) for the chiral condensate. The calculated first iteration correction modifies the choice of parameters by the following modification of the chiral-condensate formula:

$$
c=-\left(\frac{m^{*}}{2 g^{*}}\left[1+r\left(\xi^{*}\right)\right]\right)^{1 / 3} .
$$

(Accordingly, (43) and (45) are the same with the substitution $m \rightarrow m^{*}, g \rightarrow g^{*}, \kappa \rightarrow$ $\kappa^{*}, \xi \rightarrow \xi^{*}$.)

This modified choice of parameters gives:

At $c=-200 \mathrm{MeV}: \xi^{*} \cong 0.56, m^{*} \cong 420 \mathrm{MeV}, \kappa^{*} \cong 0.86$; the condensate correction is $7 \%$;

At $c=-250 \mathrm{MeV}: \xi^{*} \cong 0.3, m^{*} \cong 380 \mathrm{MeV}, \kappa^{*} \cong 0.39$; the condensate correction is $13 \%$.

Upon comparison of the given values with the ones above, the modification of the parameter choice with formula (46) diminishes the relative variation of the condensate, i.e., stabilizes the situation. It is a consequence of the positivity of the principal (pion) correction to the leading-approximation condensate.

\section{Conclusion}

According to the results obtained, the analytically regularized NJL model gives us simple closed formulae not only for the scalar amplitudes and the pion decay constant, but also for the pion contribution to the chiral condensate. As it follows from our results, in the analytically regularized NJL model, this pion contribution is significant and should be taken into account with a choice of physical values of the model parameters. At the same time, the presented analysis demonstrates that in the analytically regularized NJL model, for physical values of the parameters, taking into account nextto-leading order of the mean-field expansion, does not lead to any pathologies such as

a disappearance of the DCSB order parameters or of the Goldstone boson in the spirit of ref.[15, 16].

\section{Acknowledgment}

Authors are grateful to Profs. S.A. Hadjiev and K.G. Klimenko for useful discussions. 


\section{References}

[1] Y. Nambu and G. Jona-Lasinio: Phys. Rev. 122 (1961) 345

[2] T. Eguchi and H. Sugawara: Phys. Rev. D 10 (1974) 4257

K. Kikkawa: Prog. Theor. Phys. 56 (1976) 947

H. Kleinert: "Hadronization of quark theories", In: A. Zichichi (Ed.): Understanding the Fundamental Constituents of Matter, Plenum Press, New York, 1978, p. 289

D. Ebert and M.K. Volkov: Z. Physik C 16 (1983) 205

[3] T. Hatsuda and T. Kunihiro: Phys. Lett. B 145 (1984) 7

V. Bernard, U.-G. Meissner and I. Zahed: Phys. Rev. D 36 (1987) 819

A.S. Vshivtsev, V.Ch. Zhukovsky and K.G. Klimenko: JETP 84 (1997) 1047

D. Ebert, K.G. Klimenko, M.A. Vdovichenko and A.S. Vshivtsev: Phys. Rev. D

61 (2000) 025005

D. Ebert and K.G. Klimenko: hep-ph/0305149

[4] S.P. Klevansky: Rev. Mod. Phys. 64 (1992) 649

[5] T. Hatsuda and T. Kunihiro: Phys. Reports 247 (1994) 221

[6] P.P. Domitrovich, D. Bückers and H. Müther: Phys. Rev. C 48 (1993) 413

[7] E. Quack and S.P. Klevansky: Phys. Rev. C 49 (1994) 3283

D. Ebert, M. Nagy and M.K. Volkov: Yad. Fiz. 59 (1996) 149

[8] D. Blaschke et al.: Phys. Rev. C 53 (1996) 2394

M. Oertel, M. Buballa and J. Wambach: Nucl.Phys. A676 (2000) 247

[9] E. Babaev: Phys.Rev. D 62 (2000) 074020

M. Huang, P. Zhuang and W. Chao: Phys. Lett. B 514 (2001) 63

[10] G. Ripka: Nucl. Phys. A 683 (2001) 463

R.S. Plant and M.C. Birse: Nucl. Phys. A 703 (2002) 717

[11] S. Krewald and K. Nakayama: Ann. of Phys. 216 (1992) 201

[12] A.E. Radzhabov and M.K. Volkov: hep-ph/0305272

[13] V.E. Rochev: J. Phys. A 30 (1997) 3671

V.E. Rochev and P.A. Saponov: Int. J. Mod. Phys. A 13 (1998) 3649

V.E. Rochev: J. Phys. A 33 (2000) 7379

[14] V.E. Rochev: "An approach to approximate calculations of Green functions", In: B.B. Levchenko and V.I. Savrin (Eds.): Proceedings of XIV International Workshop on High Energy Physics and Quantum Field Theory (QFTHEP'99, Moscow 1999), MSU-Press, Moscow, 1999, p. 572

[15] H. Kleinert and B. Van den Bossche: Phys. Lett. B 474 (2000) 336

[16] T. Fujita, M. Hiramoto and H. Takahashi: hep-th/0306110 